\title{
EBSD at High Temperatures in Metals and Minerals
}

\author{
Dave Prior, Gareth Seward, Michel Bestmann, Sandra Piazolo and John Wheeler \\ Department of Earth Sciences, Liverpool University, L693GP, UK
}

EBSD is now accepted as a key tool for quantifying microstructures and textures in metals, ceramics and rocks. The scientific aim of much of this work is to understand the processes by which microstructures, textures and resultant physical properties evolve. Generally we are limited to looking at the microstructures and textures that are frozen-in after a material has undergone some kind of processing. Viable explanations of what has happened in the material are those that can explain the frozen-in microstructures and textures. Being able to watch microstructures and textures as they evolve provides a much better set of constraints upon the processes that operate in materials. The CamScan X500 crystal probe was built with the aim of conducting high temperature experiments in-situ in an SEM and quantifying microstructural and textural changes during those experiments using EBSD and imaging [1,2]. In this talk we will show some of the results of experiments in metals and in rock forming minerals[3]. We will focus on the results of phase transformation experiments in titanium and recrystallization experiments in rock salt.

During heating, at $\sim 882^{\circ} \mathrm{C}$, the crystal structure of Ti transforms from hexagonal close packed (HCP $-\alpha$ ) to body centred cubic (BCC - $\beta$ ), the opposite is observed when cooling from above $882^{\circ} \mathrm{C}$. Historically, direct observation of the $\mathrm{BCC}$ phase has been limited due to the high transformation temperature, and the fact that the BCC phase cannot be 'frozen in' metastably by quenching to lower temperatures. During an in-situ experiment we are able to observe the 'transient' microstructure and crystallography (Fig. 1b-d). We can measure the relationship between $\alpha$ and $\beta$ phases, and confirm that the first formed $\beta$ grains have the Burgers orientation relationship (OR) $\left(<0001>_{\text {hcp }} / /<110>_{\text {bcc }}\right.$ and $<11-20>_{\text {hcp }} / /<111>_{\text {bcc }}$ ) during the up temperature transformation (Fig. 1i). The $\beta$ grains are observed to have a lath shape morphology within the parent $\alpha$ grains (Fig. 1f), this together with the specific OR relationship implies a shear dominated (martensitic) transformation mechanism. There is a change in morphology of $\beta$ phase from lath shape to equiaxed grains and an accompanying order of magnitude increase in grainsize (Fig. 1g). Since the OR can only exist between a parent and daughter grain (i.e. the OR cannot be maintained with a randomly oriented neighbour) the observations suggest that grain growth processes may play a significant role in modifying the final $\beta$ texture and that rapid grain growth is triggered by the phase transformation.

Recrystallization is an important process in metals and minerals. Dry deformed rock salt samples were heated to temperatures between 350 and $410^{\circ} \mathrm{C}$. Inter- and intracrystalline processes were observed using both secondary electron imaging and electron backscatter diffraction mapping techniques. Grain boundary migration (GBM) was observed between substructured grains. During GBM some unexpected features were observed. Often the growing grain exhibits new low angle boundaries in the swept area and in some cases the existing substructure of the growing grain is continued in the swept area. More rarely (Fig. 2) substructures within the swept area develop into a low angle boundary within the growing grain. These data are not explicable with existing grain boundary migration models. The observations imply that general grain boundaries have a structure that allows structural features of the 'dying' grain to be transferred to the growing grain.

[1] G.G.E. Seward et al., Scanning. 24, (2002) 232-240. 
[2] The instrument was funded by the Higher Education Council for England (JR98LIPR) and Obducat CamScan Ltd, CamScan House, Pembroke Avenue, Waterbeach, Cambridgeshire, CB5 9PY UK.

[3] Funded by the University of Liverpool (RDF 4093) and the NERC (NER/A/S/2001/01181).

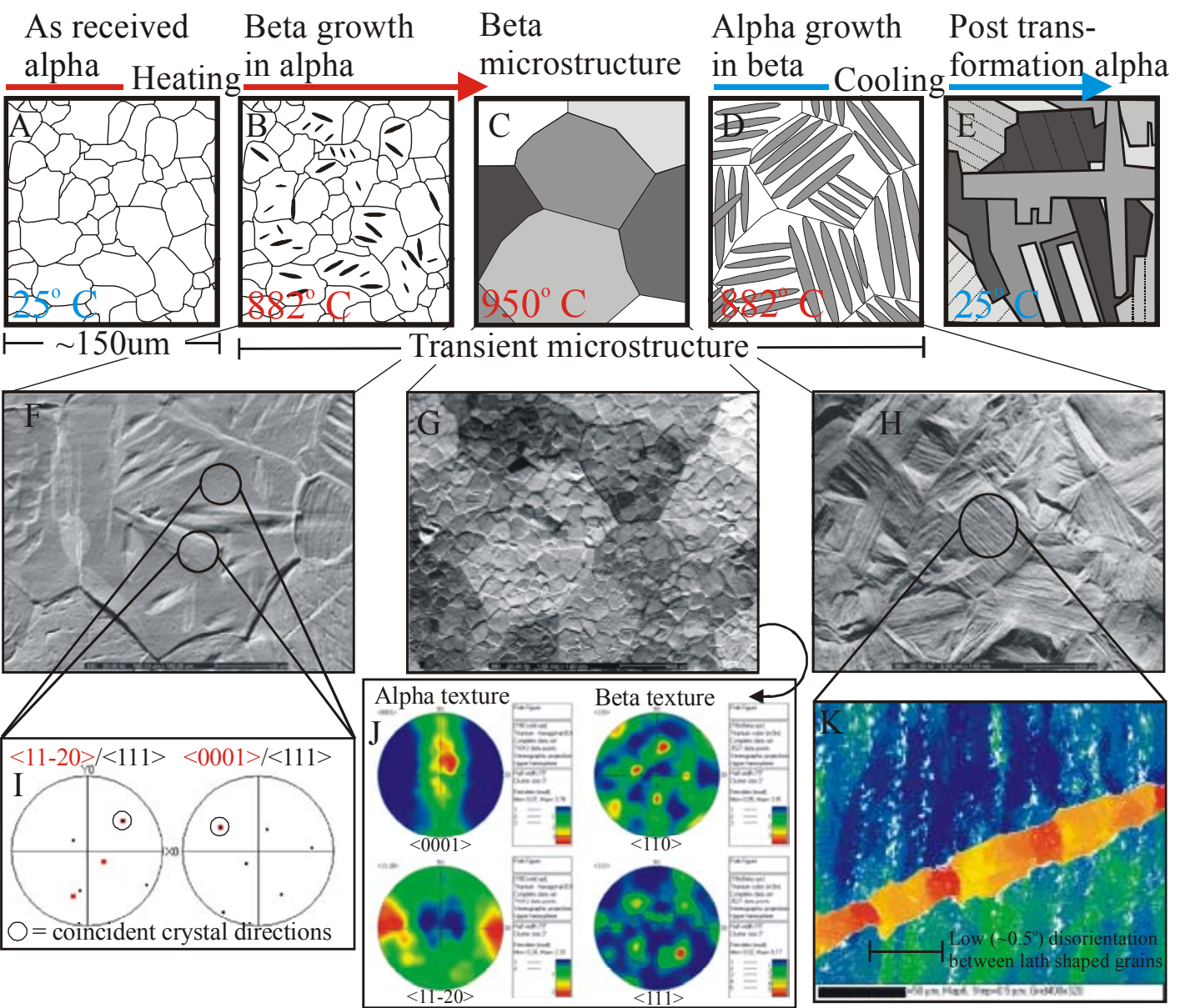

Fig 1a-k. (a)-(e) a schematic illustration of the microstructural development of Ti during heating to above the HCP-BCC phase transformation and subsequent cooling. (f)-(h) SEM micrographs of transient microstructures during an in-situ heating cycle. (i) pole figure indicating the Burgers OR between $\alpha$ and $\beta$ Ti as laths of the $\beta$ phase nucleate and grow in an $\alpha$ grain. (j) crystallographic texture pole figures comparing as received $\alpha$ texture and $\beta$ texture after transformation. (k) Lath structures post $\beta-\alpha$ transformation, separated by low angle grain boundaries.
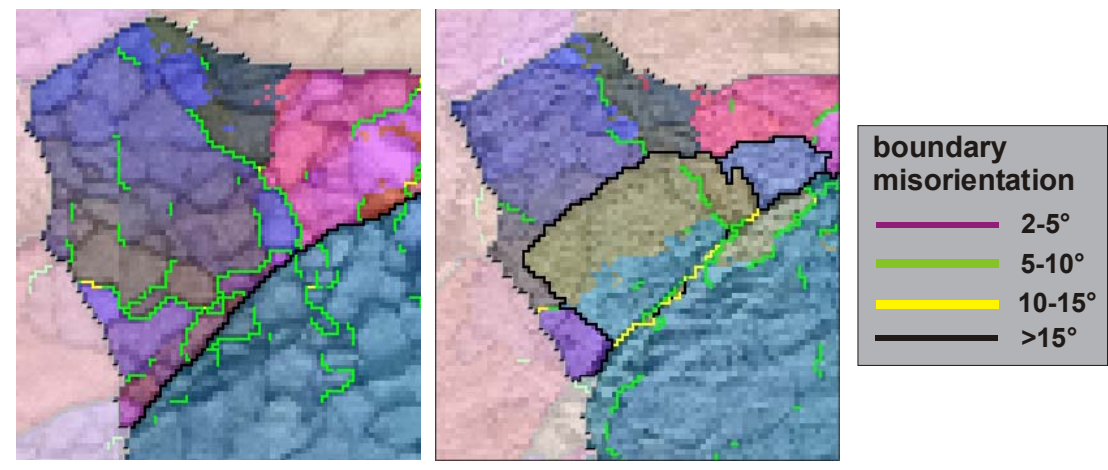

Fig 2. Two stages in the development of microstructure during heating of deformed polycrystalline rock salt. 